\title{
Estimation of Faults in DC Electrical Power System
}

\author{
Dimitry Gorinevsky*†, Stephen Boyd ${ }^{\dagger}$, and Scott Poll ${ }^{\ddagger}$
}

\begin{abstract}
This paper demonstrates a novel optimizationbased approach to estimating fault states in a DC power system. The model includes faults changing the circuit topology along with sensor faults. Our approach can be considered as a relaxation of the mixed estimation problem. We develop a linear model of the circuit and pose a convex problem for estimating the faults and other hidden states. A sparse fault vector solution is computed by using $l_{1}$ regularization. The solution is computed reliably and efficiently, and gives accurate diagnostics on the faults. We demonstrate a real-time implementation of the approach for an instrumented electrical power system testbed at NASA. Accurate estimates of multiple faults are computed in milliseconds on a PC. The approach performs well despite unmodeled transients and other modeling uncertainties present in the system.
\end{abstract}

\section{PROBlem}

We consider a DC (direct current) electric circuit with sources, loads, and switching elements. Voltage and current measurements are available at certain circuit locations. The problem is to estimate, from the measurements, the fault states of the circuit. The faults are defined as the deviations from the nominal state. The source voltages or loads can differ from their nominal values; voltage and current sensors can be faulty; assumed open/closed states of relays and breakers might differ from their actual states; short-circuit and open-circuit faults are possible, including shorts to the ground. We assume that several faults might be present simultaneously, however, it is known that most of the potential faults are not present.

Let $y \in \Re^{N_{m}}$ be a vector of observations (measurements). The fault states are described by a vector $f \in \Re^{N_{f}}$. The problem is: given the observations $y$, estimate faults $f$. The states of the circuit (e.g., voltages and currents) are described by a vector $x \in \Re^{N_{s}}$ and have to be estimated along with $f$.

Section III below describes a circuit model of the form

$$
\begin{aligned}
& 0=A x+B f+\xi, \\
& y=C x+D f+\eta,
\end{aligned}
$$

where matrices $A, B, C, D$ have appropriate dimensions. The process noise vector $\xi \in \Re^{N_{s}}$ describes the modeling error; the measurement noise $\eta \in \Re^{N_{m}}$ describes the observation error. State equation (1) and observation equation (2) are similar to linear state space model commonly used in estimation, except the model (1), (2) is static.

\footnotetext{
This work was supported by NASA Grant NNX07AEIIA

* Mitek Analytics LLC, Palo Alto, CA 94306, dimitry@mitekan.com

$\dagger$ Information Systems Laboratory, Department of Electrical Engineering, Stanford University, Stanford, CA 94305, \{gorin,boyd\}@stanford.edu

${ }^{\ddagger}$ NASA Ames Research Center, Moffet Field, CA 94035, scott.poll@nasa.gov
}

We estimate the faults $f$ and the states $x$ by solving a quadratic programming (QP) problem of the form

$$
\text { minimize } \frac{1}{2}\|A x+B f\|_{Q}^{2}+\frac{1}{2}\|C x+D f-y\|_{R}^{2}+\lambda^{T}|f|,
$$

where $\|z\|_{Q}^{2}=z^{T} Q z ; Q, R$ are positive definite matrices; $|f|$ is the component-wise absolute value; $\lambda$ is a vector with positive components. The last term provides a weighted $l_{1}$ penalty for the unknown components of vector $f$.

Optimization-based estimation of electrical power systems is well established area, e.g., see [1], [19] for an overview, more discussion is in Section II. The novelty of the formulation (3) is that the faults $f$ are introduced in a structured way separately from the states $x$. Quadratic penalty is used for mismatches $\xi, \eta$ in model (1), (2), which are usually small; an $l_{1}$ penalty is used for the fault vector $f$, which is usually large and sparse. We demonstrate that such formulation allows for efficient and accurate estimation of the faults. Work on relaxed mixed estimation related to the proposed approach is discussed in [29], [30].

Problem (3) can be interpreted as Bayesian Maximum A posteriori Probability (MAP) estimation of $x$ and $f$ given $y$. The MAP formulation assumes Gaussian noises $\xi \sim N\left(0, Q^{-1}\right)$ and $\eta \sim N\left(0, R^{-1}\right)$ in (1), (2) yileding the quadratic terms in (3). A Laplacian prior distribution assumed for the fault vector $f$ yields the $l_{1}$ term. No prior is assumed for the state $x$.

The main benefit of using $l_{1}$ regularization in (3) is that for properly selected weights $\lambda$ the solution vector $f$ is sparse. See [6], [9], [27], [28] for the recent work and further references on sparse solutions using $l_{1}$ regularization. Another advantage of QP formulation (3) is the ease of including additional linear constraints. Section V includes positivity constraints for some voltages and currents. QP problem (3) can be solved in milliseconds for hundreds of states, using modern interior-point methods, see [5].

Today electric utilities use EMS (Energy Management Systems) to monitor, control, and optimize the transmission and generation facilities. Optimization-based estimation is presently used for power systems monitoring, see [19], [1]. On-line QP optimization is also used in Model Predictive Control systems broadly employed in industry, see [24].

\section{Motivation}

There is a large body of prior work in diagnostics of electric power systems. Much work is focused on specific electric power system units and elements, such as electric machines, motors, generators, inverters, batteries, solar cells, relays, and other. Work on diagnostics of power distribution 
systems is primarily focused on AC (alternating current) systems, e.g., [8].

A few papers consider large vehicle electric systems: integrated diagnostics of international space station is discussed in [11]; diagnostics of marine vehicle power system in [16]; integrated diagnostics and prognostics of aircraft electric system in [12]. In most of this work, AI-type computational reasoning is used.

Our formulation is related to the approaches in optimization-based estimation of power system state that were first introduced in 1969, see [4], [2] and the books [1], [19] for an overview. The state vector is determined by least squares fit of the nonlinear equations relating the state variables (bus voltage magnitudes and phase angles) and the measurements (voltage and current magnitudes). The problems are generally nonconvex and an efficient solution with global convergence is difficult to achieve.

Some of the power system estimation work explicitly considers outliers in the measurements data (an equivalent of the sensor fault) and unknown states of the circuit breakers (might be caused by a fault). Using $l_{1}$ model fit error instead of the quadratic error for countering and detecting bad measurement data is discussed in [10], [14], also see [2], [4]. The papers [25], [13], [7] look into the problem of determining network topology errors. In [25], [13], mixed integer problems for determining topology are formulated. The cited papers pursue nonconvex optimization problems.

Using GPS for time synchronization made possible accurate phase measurements and harmonic state estimation of power systems, e.g., see [17]. Harmonic models are linear, which makes them close to the models in this paper. An approach using $l_{1}$ penalty in harmonic source estimation is discussed in [15].

This paper considers a DC power system with linear constitutive equations (1), (2). We show how the fault parameters can be chosen to preserve the linearity, including the topology changing faults. Convex optimization problem (3) can be solved using standard QP software. Our approach can be extended to fault estimation in harmonic power system analysis, where the equations are linear.

We implement the approach for the ADAPT EPS testbed. This testbed was developed and is maintained at NASA ARC as an experimental platform for research in integrated systems health management, diagnostics, and prognostics. More detail on ADAPT can be found in [23]. Earlier work in ADAPT diagnostics was based on AI-type reasoning and other heuristics, see [3], [18]. The proposed optimizationbased estimation approach works very well for ADAPT. Multiple faults are diagosed correctly in milliseconds.

\section{MODEL}

This section formulates a linear model of the form (1), (2) for a DC circuit.

\section{A. State equations}

We formulate the Sparse Tableau Analysis (STA) equations of the nodal DC circuit in a standard way, e.g., see
[22]. STA models are the core of most standard small-signal linear analysis of electric circuits. This section extends the STA to include a linear model of the faults. We consider several types of faults. Some of them or all of them might be present and need to be considered.

The circuit has $N_{N}$ nodes; each node has a voltage $e_{k}$ with respect to the ground. The circuit contains $N_{B}$ branches, each has current $j_{l}$ and voltage drop $v_{l}$. The signs of the currents and the voltage drops are relative to the directions of respective branches (graph edges). The incidence matrix $G \in \Re^{N_{N}, N_{B}}$ has entries $g_{k l}=1$ if the branch $l$ leaves the node $k, g_{k l}=-1$ if it enters node $k$, and $g_{k l}=0$ otherwise. The STA equations comprise Kirchhoff's Current Law (KCL), Kirchhoff's Voltage Law (KVL), and Branch Constitutive Equations (BCE), which are discussed below.

The KCL equations for the currents $j \in \Re^{N_{B}}$ are

$$
S_{B} G j=0,
$$

where $S_{B} \in \Re^{N_{I}, N_{N}}$ is a selection matrix that un-selects the boundary nodes of the circuit where KCL does not hold.

The KVL relates voltage drops $v \in \Re^{N_{B}}$ to the node voltages $e \in \Re^{N_{N}}$

$$
G^{T} e=v
$$

Finally, the BCE relate the voltage drop and the current.

$$
-K_{I} j+K_{V} v=f_{G},
$$

where $K_{V}$ and $K_{I}$ are diagonal matrices. A diagonal entry of $K_{V}$ could be conductance; in that case the respective entry of $K_{I}$ is unity. Alternatively, a diagonal entry of $K_{I}$ could be a branch resistance; in that case the respective entry of $K_{V}$ is unity. The components of the fault vector $f_{G}$ describe the faults in the branches.

We consider three types of faults. First, a fault of switching element $k$, which is thought open but might be actually closed, is expressed in the form (6) as

$$
-j_{k}=f_{G, k},
$$

where the fault parameter $f_{G, k}$ is a current through the switching element. The current should be zero if the switching element is open.

The fault of a switching element $k$, which is thought closed can be expressed in the form (6) as

$$
v_{k}=f_{G, k}
$$

where the fault parameter $f_{G, k}$ is a voltage drop at switching element. The voltage drop should be zero if the switching element is closed.

Finally, consider the fault of the DC load. We assume that the load resistance is known. The fault makes load current in branch $k$ to differ from what is given by BCE model (6)

$$
g_{k} v_{k}-j_{k}=f_{G, k},
$$

where $g_{k}$ is the branch conductance and $f_{G, k}$ is a load current deviation from the model, which is indicative of the fault. 


\section{B. Observation equations}

The STA equations need to be complemented by observation equations. We model sensor faults through additive offsets caused by these faults.

We assume that the observations include the currents $j_{\text {meas }} \in \Re^{M_{I}}$ measured by current sensors and voltages $e_{\text {meas }} \in \Re^{M_{V}}$ measured by voltage sensors. The respective observation equations have the form

$$
\begin{gathered}
e_{\text {meas }}=S_{V} e+f_{V}, \\
j_{\text {meas }}=S_{I} j+f_{I},
\end{gathered}
$$

where $f_{V}$ and $f_{I}$ are fault offsets, $S_{V}$ and $S_{I}$ are selection matrices (only some nodes and branches are instrumented).

The known voltage sources $e_{\text {srce }} \in \Re^{M_{S}}$ and grounded nodes $e_{\text {grnd }} \in \Re^{M_{G}}$ (we assume that $e_{\text {grd }}=0$ ) are counted among the observations. Though no on-line voltage sensing might be available for the source voltages, there might be an off-line knowledge of these. The respective observation equations have the form.

$$
\begin{aligned}
e_{\text {srce }} & =S_{S} e+f_{S}, \\
e_{\text {grnd }} & =S_{G} e,
\end{aligned}
$$

where $f_{S}$ is the fault offset, $S_{S}$ and $S_{G}$ are selection matrices. We do not consider ground voltage faults though it would be easy to add these to the model.

The voltage offset $f_{V}$, the current offset $f_{I}$, and the source (battery voltage) offset $f_{S}$ are unknown vectors of appropriate dimensions.

\section{STA model with faults}

To integrate the STA equations, introduce a state vector $x \in \Re^{2 N_{B}+N_{N}}$ and a matrix $A \in \Re^{2 N_{B}+N_{i n}, 2 N_{B}+N_{N}}$ as

$$
x=\left[\begin{array}{l}
j \\
v \\
e
\end{array}\right], \quad A=\left[\begin{array}{ccc}
S_{B} G & 0 & 0 \\
0 & I & -G^{T} \\
K_{I} & K_{V} & 0
\end{array}\right],
$$

Let $f$ be a vector of all fault parameters.

$$
f=\left[\begin{array}{llll}
f_{G}^{T} & f_{V}^{T} & f_{I}^{T} & f_{S}^{T}
\end{array}\right]^{T} \in \Re^{N_{F}},
$$

where $N_{F}=N_{B}+M_{V}+M_{I}+M_{S}$. Introduce the observation vector $y \in \Re^{M_{y}}$, where $M_{y}=M_{I}+M_{V}+$ $M_{S}+M_{G}$, and the observation matrix $C \in \Re^{M_{y}, 2 N_{B}+N_{N}}$, The combined observations can be expressed as

$$
y=\left[\begin{array}{c}
j_{\text {meas }} \\
e_{\text {meas }} \\
e_{\text {srce }} \\
e_{\text {grnd }}
\end{array}\right], \quad C=\left[\begin{array}{ccc}
S_{I} & 0 & 0 \\
0 & 0 & S_{V} \\
0 & 0 & S_{S} \\
0 & 0 & S_{G}
\end{array}\right]
$$

Consider matrix $B \in \Re^{2 N_{B}+N_{i n}, N_{F}}$ describing the fault impact on the states $x$ and matrix $D \in \Re^{N_{y}, N_{F}}$ describing the impact of the sensor faults on the observation vector $y$

$$
B=\left[\begin{array}{cccc}
0 & 0 & 0 & 0 \\
0 & 0 & 0 & 0 \\
B_{G} & 0 & 0 & 0
\end{array}\right], D=\left[\begin{array}{cccc}
0 & B_{I} & 0 & 0 \\
0 & 0 & B_{V} & 0 \\
0 & 0 & 0 & B_{S}
\end{array}\right]
$$

The faults enter equations linearly. After pulling together state equations (4), (5), (6) and observation equations (10),
(11), (12), and (6) and adding noises $\xi$ and $\eta$ we obtain the linear model of the form (1), (2). In this model $x, y, A$, and $C$ are given by (14), (16); the faults are defined by (15) and the fault-related matrices $B, D$ in (1), (2) have the form (17).

For zero noises $\xi=0, \eta=0$ the STA equations (1), (2) make a system of total $M_{y}+2 N_{B}+N_{i n}$ equations in the $2 N_{B}+N_{N}$ unknown components of vector $x$. In a special case of $M_{y}=N_{N}-N_{i n}$ (where $N_{N}-N_{i n}$ is the number of the edge nodes), the system is square and a unique solution can be found. This paper considers the redundant observation case of $M_{y} \geq N_{N}-N_{i n}$.

\section{VERIFICATION}

This section presents an application to ADAPT. We describe a model of the testbed, then discuss simulation results.

\section{A. Modeling}

The ADAPT circuit is shown in Figure 1, see [23]. The battery voltages $V_{1}$ and $V_{2}$ are assumed to be known. There are six branch resistances (loads) that are assumed to be known as well: these include two internal resistances of the batteries RB1, RB2; two DC load resistances RDC1, RDC2; and two equivalent resistances RAC1, RAC2, of the AC load branches (including the AC/DC inverters). The resistances of the circuit breakers and relays are assumed to be zero in the closed state and infinity in the open state. The circuit parameters were identified from experimental data.

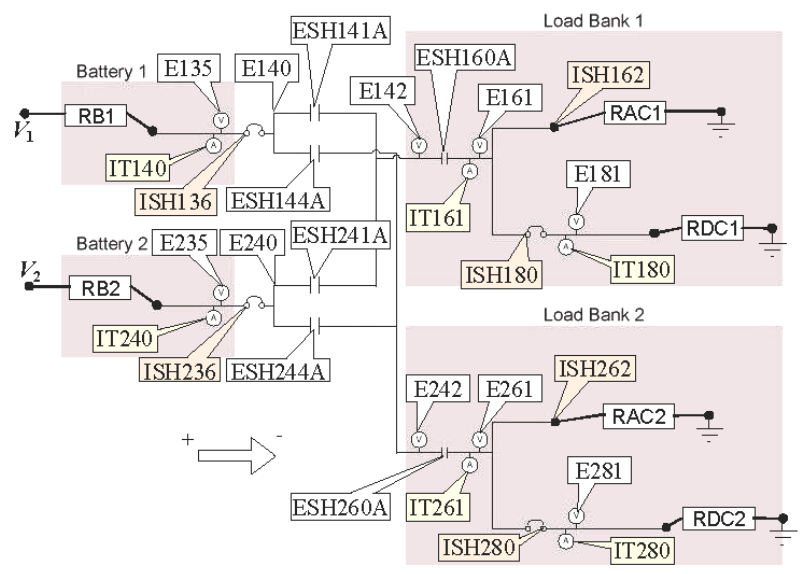

Fig. 1. Data tags for the ADAPT cicuit

The six current sensors in the circuit yield components of the current measurement vector $j_{\text {meas }}$ (11). There ten voltage sensors yield components of the voltage measurement vector $e_{\text {meas }}$ in (10).

The branch constitutive equations (6) are defined by the states of the switching elements (six relays and six circuit breakers). These open/closed states are collected from the embedded switching element sensors in ADAPT.

\section{B. Simulation}

The incidence matrix of the circuit was defined in accordance with Figure 1. The source voltages and resistances used in the simulation are summarized in Table I. 


\begin{tabular}{|c|c|c|c|c|c|c|c|}
\hline$V_{1}$ & $V_{2}$ & RB1 & RB2 & RAC1 & RDC1 & RAC2 & RDC2 \\
\hline 25.84 & 24.83 & 0.1 & 0.1 & 6 & 10 & 4 & 20 \\
\hline
\end{tabular}

ADAPT SIMULATION PARAMETERS

\begin{tabular}{|c|c|}
\hline ESH141A, ESH144A & ESH160A, ESH241A, ESH244A, ESH260A \\
\hline open & TABLE II closed \\
\hline
\end{tabular}

BASIC RELAY CONFIGURATION IN THE SIMULATION

The simulations used the STA model (1), (2), (14), (16), (15), (17) with zero noises $\xi$ and $\eta$. In simulation, current and voltage measurements were ignored by assuming $M_{I}=0$, $M_{V}=0$, and eliminating the vectors $j_{\text {meas }}, e_{\text {meas }}$, from (11), (10). The resulting system of equations has $2 N_{B}+$ $N_{N}=54$ states $x$ and $2 N_{B}+N_{i n}+M_{S}+M_{G}=54$ equations, where $N_{B}=18, N_{N}=18, N_{i n}=12, M_{S}=2$, and $M_{G}=4$. The $M_{S}$ source voltages and $M_{G}$ ground voltages (6 at all) define $N_{N}-N_{i n}=6$ boundary conditions. For given switching element states, the resulting square system was solved to determine the state vector $x$.

The switching element states define the BCE matrices $K_{I}$ and $K_{V}$ in (6), (14). The basic switching element configuration in the simulation is shown in Table II. All breakers are assumed closed. The relays are configured to connect Battery 2 to all DC and AC loads; Battery 1 is disconnected from the loads.

The simulation input is the fault state $f$, which we generated as a sparse random vector. The switching element faults were applied by inverting the respective open/closed states before calculating the BCE matrices $K_{I}, K_{V}$, and the STA matrix $A$ (14) in accordance with (7), (8).

The load faults were applied by modifying the respective load resistances in BCE (6). The resistances shown in Table I were modified by a given percentage in the range from $50 \%(-100 \%$ corresponds to a short circuit) to $+50 \%$. The source faults were applied by modifying $e_{s r c}$ in (16). The source voltages shown in Table I were modified by a given percentage in the range from $-50 \%$ ( $-100 \%$ corresponds to zero battery voltage) to zero (no change in the voltage).

The current and voltage sensor measurements at the simulation output were modified by adding offsets proportional to the respective fault magnitudes. The fault offsets for current sensors were distributed in the range from $-1 \mathrm{~A}$ to $1 \mathrm{~A}$. The fault offsets for the voltage sensors were distributed in the range from $-12 \mathrm{~V}$ to $12 \mathrm{~V}$.

In the simulations, all parametric faults were constrained to be at least $20 \%$ of the respective maximum magnitude. This avoids small faults that are below the noise level.

\section{Diagnostics of simulated data}

The diagnostics algorithm uses model (1), (2). The main differences with the simulation model are as follows.

- The diagnostics model includes faults. The fault model is described by (15), (17).
- In the diagnostics model, the switching elements are assumed to be in the nominal state. If a fault is present, the actual state differs from the nominal.

- Observations (2), (16) in the diagnostic model are the currents and the voltages distorted by noise $\eta$.

- The observation and process noises $\xi$ and $\eta$ in the diagnostic model (1), (2) are described through the inverse covariance matrices $R$ and $Q$.

The dimension of fault vector $f(15)$ is $N_{F}=N_{B}+$ $M_{V}+M_{I}+M_{S}=38$. The fault estimates were computed by solving QP problem (3) and then thresholding the absolute values of the fault vector $f$.

The problem (3) is transformed into a standard form of a QP problem with 130 decision variables. The solution using Mosek, see [20], takes about $20 \mathrm{msec}$ on a $2 \mathrm{Ghz}$ Wintel laptop computer. This is suitable for real-time implementation and much faster than $0.5 \mathrm{sec}$ sampling time for ADAPT data collection.

In the simulation experiments, a series of fault patterns were seeded (input into the simulation) to generate the sensor data. The estimation algorithms are then applied and the diagnosis of the fault state is compared to the seeded faults. In great majority of the simulation runs, the estimation algorithms diagnose the seeded faults exactly. We will focus on the cases when the diagnosis was inexact.

The first series of tests was to seed all faults, one fault at a time. There were 5 imperfect diagnoses encountered among the 38 total cases, one case for each fault component. While imperfect, these diagnoses were not, strictly speaking, incorrect. The imperfect diagnoses are discussed in Table III.

In the second series of tests two faults were seeded at a time. About 200 two-fault cases were randomly generated out of the entire set of the $38 \cdot 37 / 2=703$ double fault cases. In a majority of cases, a perfect diagnosis was achieved. Each time one of the five faults from Table III was encountered, the diagnosis was imperfect as described in the table. In addition to that, combinations of two faults introduce new ways for the diagnostic algorithm to be in error that are listed in Table IV. While imperfect, most of these diagnoses are not incorrect.

1) Circuit breaker of a disconnected battery fails open. The currents do not change, but disconnected voltage sensor now floats. This is misdiagnosed for voltage sensor fault.

2) Load circuit breaker fails open. This is misdiagnosed for an offset of the load current sensor, which would yield the same measurements. The faulty part of the circuit is pointed out correctly.

3) Same as 2, but for a different load branch.

4) Fail closed relay is diagnosed correctly. In addition to that, two more spurious faults are reported. The observed change in the current balance could be attributed to each of the three reported faults.

5) Battery output voltage is offset. The fault is detected correctly, but there is a false positive. The battery circuit breaker is misdiagnosed failing open. No current flows through the CB and it failing open cannot be excluded based on the data observed. See \# 1 for more explanation.

TABLE III

SINGLE FAULT DIAGNOSTICS ERRORS 
1) A failed circuit breaker or relay are diagnosed correctly. A second fault in the circuit part unpowered by the first fault remains undetected.

2) A fail closed relay creates a parallel connection of the two batteries This fault is diagnosed correctly. A second relay failure is misdiagnosed because the parallel connection of the batteries makes the currents through them very sensitive to the (close) battery voltages and (small) internal resistances.

3) Resistances of the two DC loads are offset, but only one of the two faults is recongnized. The second fault is below the threshold. Decreasing the $l_{1}$ regularization penalty would allow detecting the second fault but lead to false positives in other cases

TABLE IV

TWO-FAULT DIAGNOSTICS ERRORS

In summary, the proposed approach works quite well in simulation. In majority of the cases the diagnosis is perfect. In great majority of all cases, the problematic circuit branch is diagnosed correctly. The remaining imperfect diagnoses make for less than $5 \%$ of all cases and are caused by high or low problem sensitivities to some of the fault parameters.

The ADAPT circuit includes DC/AC inverters and AC loads. The inverter together with the AC load circuit can be modeled through an equivalent DC resistance. Such linearized model of inverter can be obtained by fitting experimental data on inverter draw current and supply voltage. The suitability of such a simplified inverter model is confirmed by the experimental results.

\section{VALIDATION}

The ADAPT testbed is an instrumented EPS with two sets of computer controls. The data from the first set are called "Observer" data and are used for testing diagnostic algorithms. These data were used in this work. The second, independent set of controls, known as "Antagonist" is employed in creating fault conditions for the Observer. These controlled fault conditions are known and used as a reference when evaluating diagnostic method performance. More detail on ADAPT can be found in [23].

Once verified in the simulation, the algorithms were integrated with ADAPT and validated in experiments.

One data set used for the algorithm testing is displayed in Figure 2. The data was collected at $2 \mathrm{~Hz}$ rate $(0.5 \mathrm{sec}$ interval). In the experiment, all loads are initially unpowered. Then relays EY244A and EY260A close to connect Battery 2 and to power AC Load 2. After a transient, the DC/AC Inverter 2 powers the AC load nominally. After several seconds, circuit breaker ISH262 fails (opens). This disconnects the load. The faulty circuit breaker sensor continues to show that it is closed. The described scenario is complemented by a voltage sensor fault (offset ramp). Figure 2 shows 9 plots of selected observed signals, those with a transient and those later found faulty. The remaining 27 channels out of the 36 monitored show constant values, e.g., battery voltages or circuit breaker states (all closed) and are not plotted.

Though the data contains transients, the diagnostic algorithm assume a steady state model at each step. The steady-state model used for diagnosis neglects the transients.
E140: BATT 1 CB OUTPUT VOLTAGE E242: LOAD 2 BATT OUTPUT VOLTAGE
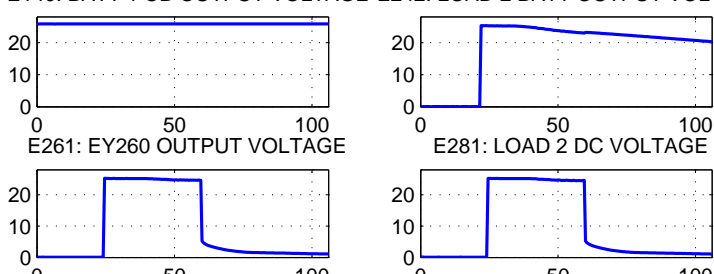
IT240: BATT 2 OUTPUT CURRENT
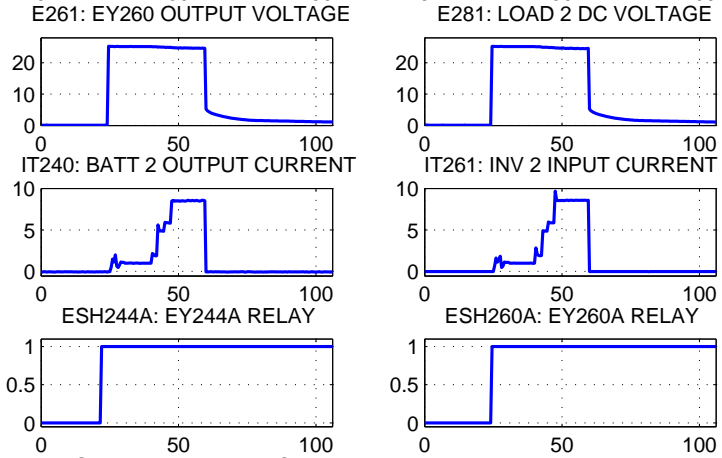

ESH260A: EYT6OA RELAY

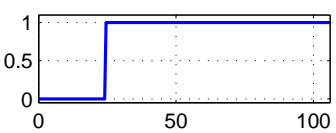

ISH262: INV 2 INPUT CB

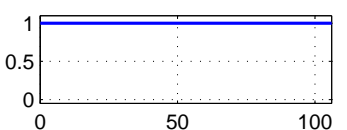

Fig. 2. Observed experimental data

E140: BATT 1 CB OUTPUT VOLTAGE E242: LOAD 2 BATT OUTPUT VOLTAGE
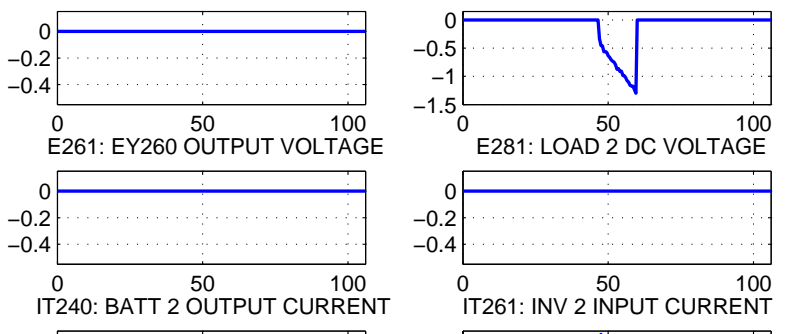

IT261: INV 2 INPUT CURRENT
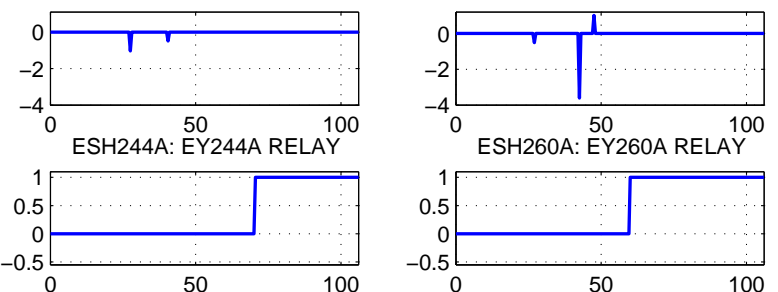

ISH262: INV 2 INPUT CB
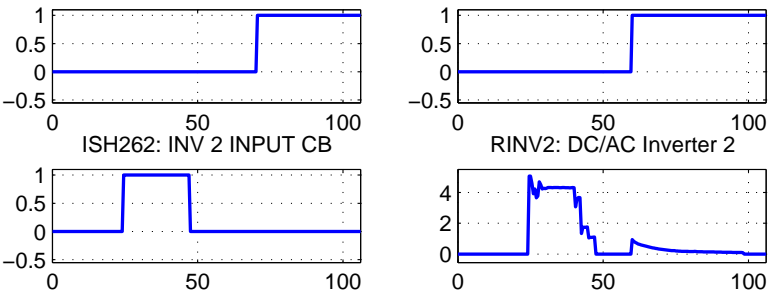

Fig. 3. Estimated fault states for the experimental data

Despite that, the algorithms are able to detect and diagnose the seeded faults. The results are illustrated in Figure 3, which shows the fault estimates corresponding to the signals in Figure 2. The diagnosis is perfect with two exceptions. First, the inverter warm-up transient is diagnosed as a fault. Second, it misses the sensor fault in the circuit branch that becomes unpowered.

The inverter model assumes a steady-state condition. However, at the start of the inverter transient at 27.5 seconds, the inverter takes approximately 6 seconds before power appears at the output. Then, it takes an additional 14 seconds before all the loads are turned on and the equivalent resistance 


\begin{tabular}{ll}
\hline \hline Time: Event & Description \\
\hline $\mathrm{t}=24:$ Control action & $\begin{array}{l}\text { Relays EY244A and EY260A are closed to } \\
\text { power the load }\end{array}$ \\
\hline $\begin{array}{l}\mathrm{t}=27.5: \text { Start of INV2 } \\
\text { transient }\end{array}$ & $\begin{array}{l}\text { INV2 Input CD ISH262 is suspected open; } \\
\text { INV2 Load is suspected to be off-nominal }\end{array}$ \\
\hline $\mathrm{t}=34:$ Sensor fault oc- & $\begin{array}{l}\text { E242 voltage offset starts ramping down from } \\
\text { zero }\end{array}$ \\
\hline $\mathrm{t}=46.5:$ Sensor fault de- & $\begin{array}{l}\text { E242 voltage offset is detected during the } \\
\text { inverter transient }\end{array}$ \\
tected & Inverter reaches a steady state \\
\hline $\mathrm{t}=47.5:$ Nominal regime & $\begin{array}{l}\text { Circuit breaker ISH262 opens and cuts off } \\
\text { the branch with the DC/AC inverter load and }\end{array}$ \\
fault occurs & E242 voltage sensor \\
\hline $\begin{array}{l}\mathrm{t}=60: \text { Circuit breaker } \\
\text { fault detected, sensor } \\
\text { offset detected }\end{array}$ & $\begin{array}{l}\text { Circuit breaker ISH262 failure is detected; } \\
\text { stops being detected }\end{array}$ \\
\hline $\mathrm{t}=65.5:$ Relay fault de- \\
tected, sensor fault un- \\
$\begin{array}{l}\text { The large offset of voltage measurement E242 } \\
\text { is now interpreted as a voltage across relay } \\
\text { of a second relay fault }\end{array}$ & $\begin{array}{l}\text { EY244A in the disabled branch indicating } \\
\text { that the relay failed open. }\end{array}$ \\
\hline \hline
\end{tabular}

TIMELINE OF EVENTS IN EXPERIMENTAL DATA AND FAULT ESTIMATION

reaches the full RAC2 value that is used in the model.

The lower right plot shows an estimate of the fault current (deviation from the nominal current) for the DC/AC Inverter 2 with the AC load. Switching of the relays and inverter excites very large current transients. These causes false positives for current sensor faults. The middle plots in Figure 3 show three false positive occurrences for one sensor and two for another, one time-sample duration each. The timeline of the events and the diagnostic estimation results with a commentary are detailed in Table V.

\section{CONCLUSIONS}

The proposed diagnostics approach works very well for the DC power system applications exemplified by ADAPT. The diagnosis is accurate when this can be expected. The algorithm has a few well-defined parameters that need to be set up and takes milliseconds to compute results. The approach has been demonstrated to work well with experimental data despite ignoring the transients and modeling a complex circuit of DC/AC inverter with a load by a single DC resistor. The approach is clearly suitable for online implementation in practical monitoring applications for electric power systems.

\section{REFERENCES}

[1] A. Abur and A. Gomez Exposito, Power System State Estimation: Theory and Implementation, New York, NY: Marcel Dekker, 2004.

[2] M.E. Baran and A. Abur, "Power System State Estimation," in J. Webster (ed.), Wiley Encyclopedia of Electrical and Electronics Engineering, 1999 John Wiley and Sons, Inc.

[3] M. Daigle, X. Koutsoukos, and G. Biswas, "An Event-based Approach to Integrated Parametric and Discrete Fault Diagnosis in Hybrid Systems," Trans. Inst. of Measurement and Control, Special Issue on Hybrid and Switched Systems, to appear.

[4] A. Bose and K.A. Clements, "Real time modeling of power networks," Proc. IEEE, Vol. 75, No. 12, pp. 1607-1622, 1987.

[5] S. Boyd and L. Vandenberghe, Convex Optimization, Cambridge University Press, 2004.

[6] E. J. Candes, J. Romberg, and T. Tao, "Stable signal recovery from incomplete and inaccurate measurements," Communications on Pure and Applied Mathematics, Vol. 59, 2006, pp. 1207-1223.
[7] K.A. Clements and A.S. Costa, "Topology error identification using normalized Lagrange multipliers", IEEE Trans. on Power Systems, Vol. 13, No. 2, pp. 347-353, 1998.

[8] E.M. Davidson, S.D.J. McArthur, J.R. McDonald, "A toolset for applying model-based reasoning techniques to diagnostics for power systems protection," IEEE Trans. on Power Systems Vol. 18, No. 2, 2003, pp. 680-687.

[9] D. Donoho, "Compressed sensing," IEEE Transactions on Information Theory, Vol. 52, No 4, 2006, pp. 1289-1306.

[10] M.R. Irving, R.C. Owen, and M.J.H. Sterling, "Power system state estimation using linear programming," Proc. IEE., Vol. 125, pp. 879$885,1978$.

[11] A. Gonzalez, R. Morris, F. McKenzie, D. Carreira, B. Gann, "Modelbased, realtime control of electrical power systems," IEEE Trans. on Systems, Man, and Cybernetics, Vol. 26, No. 4. 1996, pp. 470-482

[12] K. Keller, K. Swearingen, J. Sheahan, et al., "Aircraft electrical power systems prognostics and health management," IEEE Aerospace Conf., March 2006, Big Sky, MT.

[13] G. N. Korres and P. J. Katsikas, "Identification of Circuit Breaker Statuses in WLS State Estimator," IEEE Trans. on Power Systems, Vol. 17, No. 3, pp. 818-825, 2002.

[14] W.W. Kotiuga and M. Vidyasagar, "Bad data rejection properties of weighted least absolute value techniques applied to static state estimation," IEEE Trans. Power Appar. Syst., Vol. PAS-101, pp. 844851, 1982.

[15] H. Liao, "Power System Harmonic State Estimation and Observability Analysis via Sparsity Maximization," IEEE Trans. on Power Systems, Vol. 22, No. 1, pp. 15-23, 2007.

[16] L. Li, K.P. Logan, D.A. Cartes, S.K.Srivastava, "Fault detection, diagnostics, and prognostics: software agent solutions," IEEE Transactions on Vehicular Technology, Vol. 56, No. 4, July 2007, pp. 1613-1622.

[17] A. P. Sakis Meliopoulos, F. Zhang, and S. Zelingher, "Power system harmonic state estimation," IEEE Trans. an Power Delivery, Vol. 9, pp. 1701-1709, 1994.

[18] O.J. Mengshoel, A. Darwiche, K. Cascio, M. Chavira, S. Poll, and S. Uckun, "Diagnosing Faults in Electrical Power Systems of Spacecraft and Aircraft," 20th Innovative Applications of Artificial Intelligence Conference (IAAI-08), July 2008, Chicago, IL.

[19] A. Monticelli, State Estimation In Electric Power Systems: A Generalized Approach, Boston: Kluwer, 1999.

[20] MOSEK ApS, The MOSEK Optimization Tools Version 5.0. Optimization Tool Manual., 2007. Available: www.mosek.com.

[21] F. Muzi and G. Sacerdoti, "A numerical approach to the diagnostics of electrical distribution networks," IEEE Power Engineering Society General Meeting, June 2007

[22] L.T. Pillage, R.A. Rohrer, C. Visweswarian, Electronic Circuit and System Simulation Methods (SRE), McGrawHill, 1998

[23] S. Poll, A. Patterson-Hine, J. Camisa, D. Garcia, et al., "Advanced Diagnostics and Prognostics Testbed", 18th International Workshop on Principles of Diagnosis, pp. 178-185, May 2007.

[24] S.J. Qin and T.A. Badgewell, "An overview of industrial model predictive control technology," In Chemical Process Control - V, volume 93, no. 316, pages 232-256, AIChe Symposium Series American Institute of Chemical Engineers, 1997

[25] H. Singh and F.L. Alvarado "Network Topology Determination using Least Absolute Value State Estimation," IEEE Trans. on Power Systems, Vol. 10, No. 3, pp. 1159-1165, 1995

[26] R. Spyker, D.L. Schweickart, J.C. Horwath, L.C. Walko, D. Grosjean, "An evaluation of diagnostic techniques relevant to arcing fault current interrupters for direct current power systems in future aircraft," Electrical Insulation Conference and Electrical Manufacturing Expo, pages 146-150, Oct. 2005.

[27] R. Tibshirani, "Regression shrinkage and selection via the lasso," Journal of the Royal Statistical Society. Series B (Methodological), Vol. 58, No. 1, 1996, pp. 267-288.

[28] J. Tropp, "Just relax: convex programming methods for identifying sparse signals in noise," IEEE Transactions on Information Theory, Vol. 52, No 3, 2006, pp. 1030-1051.

[29] A. Zymnis, S. Boyd, and D. Gorinevsky, Mixed state estimation for a linear Gaussian Markov model. 47th IEEE CDC, pages 3219-3226, 2008

[30] A. Zymnis, S. Boyd, and D. Gorinevsky, Relaxed maximum a posteriori fault identification. Signal Processing, Vol. 89, No. 6, 2009, pp. 989-999. 